\title{
Quantum theory of $k(\phi)$-FLRW-metrics its connection to Chern-Simons-models and the theta vacuum structure of quantum gravity
}

\author{
J. Hristov ${ }^{1, \mathrm{a}}(\mathbb{D}$ \\ ${ }^{1}$ University of Leipzig, Leipzig, Germany
}

Received: 14 April 2021 / Accepted: 3 June 2021 / Published online: 6 July 2021

(C) The Author(s) 2021, corrected publication 2021

\begin{abstract}
We show how a foliated 4-dimensional FLRWmetric becomes a gravitational instanton, if the spatial metric minimizes a three-dimensional Einstein-Hilbert action with positive cosmological constant, which is equal to the demand, that the scale factor satisfies the Bogomolny-equation, where the curvature parameter varies over the one-parameter family of hyperslices and takes the role of a potential depending on the scale factor. Additionally, we draw the connection to SO(4)-Chern-Simons theory and show how the established interpolating solutions describe the gradient flow between the minima of the vacuums of the Einstein-Hilbert action, as well as how they can be used to calculate tunnelling-amplitudes of gravitons and trivialize the calculations of path integrals in quantum gravity. All the calculations are carried out particularly for $k$ admitting a $\mathbb{Z}_{2}$-symmetry.
\end{abstract}

\section{Introduction}

We would like to describe the evolution of spacetime as a trajectory of a three-dimensional pseudo-Riemannian manifold $(\Sigma, h)$ with positive, constant scalar curvature equipped with metric tensor $h_{i j}\left(x^{k}\right)$ in a special kind of double-well potential. We assume a four-dimensional, globally hyperbolic manifold $(M, g)$ is foliated by the family $\Sigma_{t}$ under synchronous gauge.

$$
\begin{aligned}
& \beta_{i}=\mathbf{0} \\
& g_{00}=-N(t)^{2}+\beta_{k} \beta^{k} \\
& g_{\mu \nu}=\left(\begin{array}{cc}
-N(t)^{2}+\beta_{k} \beta^{k} & \beta_{j} \\
\beta_{i} & h_{i j}
\end{array}\right) \\
& h_{i j}\left(x^{k}, t\right)=\phi^{2}(t) \gamma_{i j}
\end{aligned}
$$

\footnotetext{
a e-mail: julius.hristov@web.de (corresponding author)
}

where $\beta_{i}$ is called the Shift, $N$ the lapse, $\gamma_{i j}$ is the maximally symmetric metric on the hyperslice and $\phi$ is the scale factor. $\Sigma_{t}$ is the image of the embedding

$\mathfrak{E}_{t}: \Sigma \rightarrow \mathrm{M}$

so that $\left(\Sigma, h_{t}\right)$ is isomorphic to $\Sigma_{t}$. One should be reminded of the absence of any implication, relating the parameter $t$ to clock readings so far. In this case $t$ should be understood as a flow parameter. The action consists of the Einstein-Hilbert action and the Gibbons-Hawking-York boundary term.

$$
\begin{aligned}
S\left[g_{\mu \nu}\right]= & \frac{1}{16 \pi l_{p}^{2}} \int_{M} \sqrt{g}(4) R d^{4} x \\
& +\frac{1}{8 \pi l_{p}^{2}} \int_{\Sigma_{t}} \varepsilon \sqrt{h} K d^{3} x .
\end{aligned}
$$

For the given metric, the four-curvature is of the form

${ }^{(4)} R=\frac{6}{\phi^{2} N^{3}}\left(N\left(\phi \ddot{\phi}+\dot{\phi}^{2}\right)-\phi \dot{\phi} \dot{N}+k N^{3}\right)$.

After elimination of the second derivative, through integration by parts, the total derivative is cancelled exactly by the boundary term, where the appropriate choice of $\varepsilon=-1$ was made for spacelike hypersurfaces. This leaves us with

$$
\begin{aligned}
L_{G} & =L_{E H}+L_{G H Y}=\sqrt{g}(4) R-2 \sqrt{h} K \\
& =\frac{6 \phi}{N^{2}}\left(-N \dot{\phi}^{2}+k N^{3}\right) .
\end{aligned}
$$

The Ricci-Tensor of the maximally symmetric metric is defined by

${ }^{(3)} R_{i j}=2 k \gamma_{i j}$.

We assume $k$ is a one-parameter family of the flow parameter $t \rightarrow k(t) \in[0,1]$. Although $k$ remains a constant for every maximally symmetric hypersurface as required by the Bianchi-Identity, for the foliated manifold $M$, since it varies over the set of all hypersurfaces $\Sigma_{t}, k$ becomes the dynamical 
parameter $k(t)$. Furthermore, it seems natural to assume a dependence of the curvature parameter on the scale factor. Setting $N(t)= \pm 1$ and $k(\phi)=\dot{\phi}^{2}$ in Eq. (1.5), produces a gravitational instanton, because the gravitational action (1.3) vanishes, implying that under the assumptions for the dynamics made above, $g_{\mu \nu}$ is an extreme point of $S\left[g_{\mu \nu}\right]$ and satisfies the vacuum Einstein equations:

$R_{\mu \nu}-\frac{1}{2} R g_{\mu \nu}=0$

It should therefore have a representation as a sum of (anti)self dual instantons in Yang-Mills-Theory [1]. As we will see later, this is indeed the case. Now the line element in spherical coordinates is

$$
\begin{aligned}
d s^{2}= & g_{\mu \nu} d x^{\mu} d x^{\nu}=-d t^{2} \\
& +\underbrace{\frac{\phi(t)^{2}}{1-k(\phi) r^{2}}}_{\Delta \phi} d r^{2}+\phi(t)^{2} r^{2} d \Omega .
\end{aligned}
$$

Furthermore, the requirement that $k(\phi)=\dot{\phi}^{2}$ enables a connection to field theory, because

$$
\frac{\partial \phi}{\partial t}= \pm \sqrt{k(\phi)}
$$

could be interpreted as the Bogomolny-equations, whose solutions are extreme points of the system defined by the action.

$S[\phi]=\frac{1}{2} \int d t\left(\partial_{t} \phi\right)^{2}+k(\phi)$.

In this case the scale factor $\phi$ of the metric $g_{\mu \nu}$ would be seen, as a one-dimensional field configuration and $k(\phi)$ as the potential, which would put some restraints onto $\phi$, the vacuum structure of the system and therefore on the topology of $k(\phi)$. Since we are interested in non-trivial, finite energy solutions, the homotopy class $\pi_{0}(\mathcal{V})$ of the non-connected vacuum manifold $\mathcal{V}=\left\{\phi_{0}, \dot{\phi}_{0}=0, k\left(\phi_{0}\right)=k_{\text {min }}=0\right\}$ must not be trivial $[4,14]$. Additionally, the requirement for finite energy imposes the boundary condition $\lim _{x \rightarrow \pm \infty} \phi(x)$ $=\phi_{ \pm}$onto the field, where $\left(\phi_{+}, \phi_{-}\right) \in \pi_{0}(\mathcal{V}) \times \pi_{0}(\mathcal{V})$. For a non-trivial solution $\phi_{+} \neq \phi_{-}$holds. From that one is able, to derive a stronger statement than the virial theorem for the energy of a configuration.

$E \geq\left|U\left(\phi_{+}\right)-U\left(\phi_{-}\right)\right|$

where $k(\phi)=\frac{1}{2}\left(\frac{d U(\phi)}{d \phi}\right)^{2}$. Equality requires the field configuration $\phi$ to satisfy the Bogomolny-equations [2-4]

$\frac{\partial \phi}{\partial t}= \pm \sqrt{k(\phi)}$ with boundary condition $\lim _{x \rightarrow \pm \infty} \phi(x)=\phi_{ \pm}$. It is equivalent to

$\ddot{\phi}(t)=\frac{1}{2} \frac{\partial k(\phi)}{\partial \phi}$.

The simplest vacuum structure is $\pi_{0}(\mathcal{V})=\mathbb{Z}_{2}$. We choose

$k(\phi)=R_{0}\left(1-\phi^{2}(t)\right)^{2}$

where the Planck curvature $R_{0}=\frac{1}{l_{p}^{2}}=M_{p}^{2}$ is the hight of the false minima of $k(\phi)$, which is set to 1 , so that the curvature reaches a maximum for $t=0$. By construction this would mean, that every solution $\phi$ of (1.8), trivial or nontrivial, which minimizes the action functional (1.9), is the scale factor of a four-dimensional manifold $M$ with metric $g_{\mu \nu}$ defined above, that satisfies the Einstein vacuum equations and is therefore a gravitational instanton. Observe, that if $k(\phi)=\frac{\Lambda}{3} \phi^{2}$, the manifold $(M, g)$ would be a de-Sitter, anti-de-Sitter space respectively, because

$\phi(t)=e^{ \pm \sqrt{\frac{\Lambda}{3}} t}$

where the cosmological constant acts as a spring constant (Fig. 1). For the given potential (1.12) however, the solutions are

$$
\begin{aligned}
\phi\left(t-t_{0}\right) & = \pm \tanh \left(M_{p}\left(t-t_{0}\right)\right) \\
\Delta(\phi) & =\frac{\phi(t)^{2}}{1-k(\phi) r^{2}} \\
& =\frac{\tanh ^{2}\left(M_{p}\left(t-t_{0}\right)\right)}{1-M_{p}^{2} \operatorname{sech}^{4}\left(M_{p}\left(t-t_{0}\right)\right) r^{2}}
\end{aligned}
$$

and

$$
\begin{aligned}
& \phi_{0}= \pm 1 \\
& \Delta(\phi)=1 .
\end{aligned}
$$

Interestingly, only if $r \leq l_{p}, \Delta(\phi)$ in (1.13a) admits a non-singular behaviour, although $r=l_{p}$ is clearly favoured cosmologically speaking due to the absence of a zero at the origin, as it is present for $r<l_{p}$. In this case, even though $\phi(0)=0$, a singularity is avoided because curvature and energy density stay finite due to the finiteness of the false maximum of $k(\phi)$, which is $R_{0}=1 / l_{p}^{2}$. By construction, the scale factor may take negative values but since stable systems in Lorentzian time require potentials bounded from below and because we set $k(t) \in[0,1]$, the curvature is also strictly semi-definite. The associated metrics are indeed extreme points of the action $S\left[g_{\mu \nu}\right]$ defined in (1.3)

$$
\begin{aligned}
d s^{2} & =g_{\mu \nu} d x^{\mu} d x^{\nu} \\
& =-d t^{2}+\tanh ^{2}\left(M_{p}\left(t-t_{0}\right)\right) \gamma_{i j} d x^{i} d x^{j}
\end{aligned}
$$


Fig. 1 Illustration of the manifold's scale factor $\phi(t)$ for $t_{0}=0$ as a one-dimensional field configuration interpolation between the two minima of $k(\phi)$, which is proportional to the scalar curvature

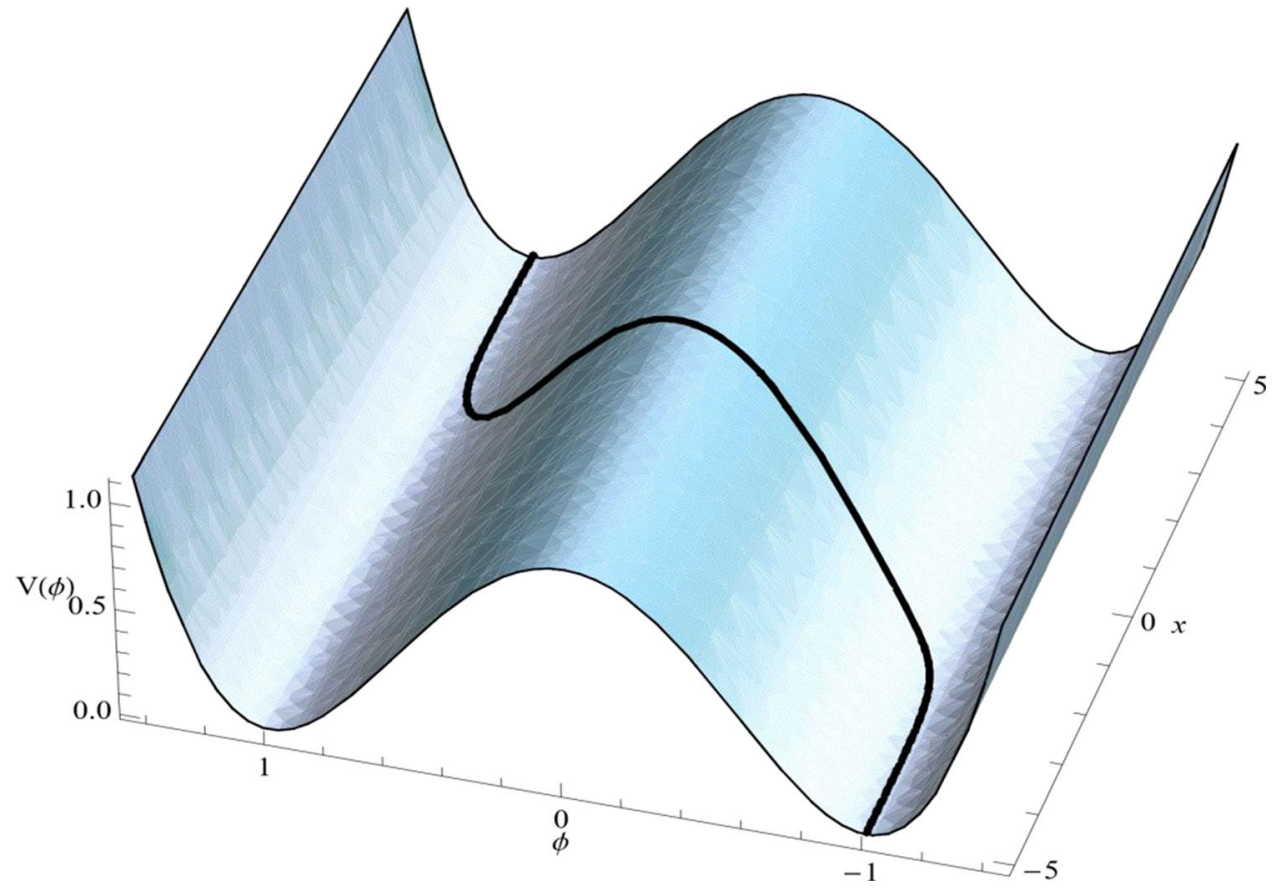

The manifolds associated with the trivial solutions of the system (1.9) are simply Minkowski spaces,

$d s^{2}=g_{\mu \nu} d x^{\mu} d x^{\nu}=-d t^{2}+\delta_{i j} d x^{i} d x^{j}$

where $\delta_{i j}=\operatorname{diag}(1,1,1)$, between which the non-trivial solution (1.13a) interpolates. This can be seen by looking at the limits of $\Delta(\phi)$ in the non-trivial sector.

$\lim _{\phi \rightarrow \pm \phi_{0}} \Delta(\phi)=\lim _{t \rightarrow \pm \infty} \Delta(\phi)=1$.

Observe furthermore, that the pseudo-Riemannian metric $h_{i j}(t)$ satisfies the Hamilton constraint of ADM-formalism

$$
\begin{aligned}
H & =\int_{\Sigma_{t}} \mathcal{H} d^{3} x \\
& =-\int_{\Sigma_{t}} N \sqrt{h}\left(K^{i j} K_{i j}-K^{2}+{ }^{(3)} R\right) d^{3} x=0
\end{aligned}
$$

because the extrinsic curvature tensor of the constant $t$ hypersufaces in the ambient spacetime is

$K_{i j}=\frac{1}{2 N} \partial_{t} h_{i j}=\frac{\dot{\phi}}{N \phi} h_{i j}$.

The trace is

$K=h^{i j} K_{i j}=\frac{3 \dot{\phi}}{N \phi}$

and

${ }^{(3)} R=h^{i j} R_{i j}=\frac{6 k}{\phi^{2}}=\frac{6 M_{p}^{2}}{\phi^{2}}\left(1-\phi^{2}(t)\right)^{2}=6 \frac{\dot{\phi}^{2}}{\phi^{2}}$.
Concluding

$K^{i j} K_{i j}-K^{2}+{ }^{(3)} R=3\left(\frac{\dot{\phi}}{N \phi}\right)^{2}-\left(\frac{3 \dot{\phi}}{N \phi}\right)^{2}+6 \frac{\dot{\phi}^{2}}{\phi^{2}}=0$.

Since the integrand of (1.17) is equivalent to the fourcurvature in (1.3), it is only another way of proving, that

$d s^{2}=g_{\mu \nu} d x^{\mu} d x^{\nu}=-d t^{2}+h_{i j}(t) d x^{i} d x^{j}$

is Einstein and defines a gravitational instanton. Returning to the example of our double-well potential, virtually every manifold of the family $\Sigma_{t}$ described by a $h_{i j}(t)$ is almost indistinguishable from a Euclidean manifold, except for those in the close vicinity of the bounce at $t_{0}$. All together this means, we associated the systems (1.3) and (1.9) to each other by turning $k$ into a dynamical parameter through a foliation of a gravitational instanton and viewing it as the potential of a one-dimensional system, of which the scale factor of the four-metric is a topological solution to. $k$ is therefore proportional to the scalar curvature and the evolution of the scale factor inside the potential of the intrinsic curvature determines the structure of the gravitational instanton. Putting everything together, corresponding to the two kink solutions (1.13a), interpolating between the minima of the potential $k(\phi)$, the associated gravitational instanton interpolates between two Minkowskian manifolds at $t= \pm \infty$, in correspondence with the trivial solutions of the one-dimensional system (1.13b). Since the wick-rotated kink is an instanton, which is the classical analogous of the quantum mechanical tunnelling effect, the question occurs, if in analogy, the expanding and con- 
tracting spacetime with inflationary periods in the vicinity of the bounce at $t=0$, described by the gravitational instanton $M$, is the classical equivalent of the graviton ground states of two Euclidean manifolds, resembling the minima of the potential of Ricci curvature, tunnelling through the potentialwell between them.

\section{Gravitational instantons and the gradient flow of the Chern-Simons invariant}

The ideas of the previous section are supported by the identification of the " $k(\phi)$-theory" with the Chern-Simons theory. The connection can be seen as follows. From (1.6) we can read of the dependence of the Ricci tensor on the scale factor in the flow equation [5]

${ }^{(3)} R_{i j}=2 \dot{\phi}^{2} \gamma_{i j}=2 \frac{\dot{\phi}^{2}}{\phi^{2}} \phi^{2} \gamma_{i j}=\frac{{ }^{(3)} R}{3} h_{i j}$

where ${ }^{(3)} R=h^{i j} R_{i j}$. Expression (2.1) is nothing less than the vacuum field equations of GR in three dimensions with a cosmological constant, which we shall denote as $\Lambda$.

$S_{E H}\left[h_{i j}\right]=\frac{M_{p}^{2}}{16 \pi} \int d^{3} x \sqrt{h}\left({ }^{(3)} R-2 \Lambda\right)$.

Variation with respect to the inverse three-metric yields

$\delta S_{E H}=\int\left({ }^{(3)} R_{i j}-\frac{{ }^{(3)} R}{2} h_{i j}+\Lambda h_{i j}\right) \delta h^{i j} d^{3} x=0$,

which is just (2.1) for

$\Lambda(\phi)=\frac{k(\phi)}{\phi^{2}}=\frac{\dot{\phi}^{2}}{\phi^{2}}$.

This means that for static solutions the cosmological constant vanishes. Since the action functional (2.2), describing three-dimensional gravity with a cosmological constant, is equivalent to the CS-theory on the three-surface $\Sigma$ [6], with the gauge group $S O(4)=S O(3) \times S O(3)$, where the spatial metric is described by $\mathfrak{g}$-valued connection one-forms $A_{i}^{a \pm}$ on a principial G-bundle, the foliated gravitational instanton $g_{\mu \nu}$, minimizing the four dimensional first-order action (1.3), while connecting its vacuums, is equivalent to a Yang-Mills instanton on $\Sigma \times \mathbb{R}$ embodying trajectories of the gradient flow of the Chern-Simons action functional with respect to the Hodge inner product metric on the bundle

$h(a, b)=\int_{\Sigma}\left\langle a, \star_{h} b\right\rangle$,

where $\langle-,-\rangle: \mathfrak{g} \otimes \mathfrak{g} \rightarrow \mathbb{R}$ is the killing form on the Liealgebra $\mathfrak{g}$. The CS-action functional is

$S_{C S}[A]=\frac{k}{4 \pi} \int_{\Sigma} \operatorname{Tr}\left(A \wedge d A+\frac{2}{3} A \wedge A \wedge A\right)$ where $A \in \Omega^{1}(\Sigma, \mathfrak{g})$ and $T r$ is in the Lie-algebra. The action is minimized for flat connections satisfying

$F=d A+A \wedge A=0$.

In the case of $S O(4)$, the differential comohology would be a spin-connection. To see the equivalence of (2.5) and (2.2), we define the gauge fields $A_{j}^{a \pm}$ to be in $\mathfrak{g}=\mathfrak{s o}(3)$ and write them as a combination of the spin connection $w_{j}^{a}$ and the dreibein $e_{j}^{a}$

$A_{j}^{a \pm}=w_{j}^{a} \pm \sqrt{\Lambda} e_{j}^{a}$.

The covariant derivative is

$D_{j}=\partial_{j}+J_{a}^{+} A_{j}^{a+}+J_{a}^{-} A_{j}^{a-}$

with $J_{a}^{ \pm}$the generators of the Lie-algebra of $S O(3)$. The CS-action is then

$$
\begin{aligned}
S_{C S}\left[A^{ \pm}\right]= & \frac{M_{p}^{2}}{32 \pi \sqrt{\Lambda}} \\
& \times \int \epsilon^{i j k}\left(A_{i}^{a \pm} \partial_{j} A_{k}^{a \pm}+\frac{1}{3} \epsilon_{a b c} A_{i}^{a \pm} A_{j}^{b \pm} A_{k}^{c \pm}\right),
\end{aligned}
$$

from which follows that

$S_{E H}=S_{C S}\left[A^{+}\right]-S_{C S}\left[A^{-}\right]$.

A trivial solution of the Bogomolny-type equation (1.6) is therefore a scale factor of a metric on $\Sigma$ in synchronous gauge, that is equivalent to a vacuum of a threedimensional CS-theory, while the non-trivial solutions are a one-parameter family of scale factors on $\Sigma$, foliating a gravitational instanton. Equivalently, Yang-Mills instantons in the temporal gauge $\mathrm{A}_{\partial_{t}}^{ \pm}=0$, regarding the fact that they are one parameter families of connections on $\Sigma$, satisfying the self-duality condition

$\frac{d}{d t} A^{ \pm}=\frac{\delta S_{C S}\left[A^{ \pm}\right]}{\delta A^{ \pm}}= \pm \star_{h} F_{A^{ \pm}} \in \Omega^{1}(\Sigma, \mathfrak{g})$

characterize the gradient flow between different CS-vacua. The above is also captured by the instanton Floer-homology [7,8]. Expression (2.11) can be viewed as the analogue to Eq. (2.1). It indicates that the family of connections $A^{ \pm}$on $\Sigma$ is an (anti)-self-dual, finite energy solution to the YangMills equations on $\Sigma \times \mathbb{R}$ with metric $h \otimes I$

$$
\begin{aligned}
S_{Y M}\left(A^{ \pm}\right) & =\int_{M=\Sigma \times \mathbb{R}} \operatorname{Tr}\left(F_{A^{ \pm}} \wedge \star F_{A^{ \pm}}\right)<\infty \\
\star F_{A^{ \pm}} & = \pm F_{A^{ \pm}}
\end{aligned}
$$

In a similar way is every solution to (2.1) a flowline of the gradient flow between vacua of (2.2) and defines a gravitational instanton in the gauge $N=1$, that is an (A)SD solution to the pure field equations of GR on $\Sigma \times \mathbb{R}$. The 
fact that two instantons $A_{j}^{a \pm}$ correspond to a single gravitational instanton is reflected by the two scale factors (kink and anti-kink) belonging to the same $g_{\mu \nu}$ and because of the decomposition $S O(4)=S O(3) \times S O(3)$. Hence, we can think of $g_{\mu \nu} \in \Gamma\left(\mathcal{T}^{0,2}(M=\Sigma \times \mathbb{R})\right)$ accordingly as the one-parameter family

$t \rightarrow h_{i j}(t)=( \pm \phi(t))^{2} \gamma_{i j} \in \Gamma\left(\mathcal{T}^{0,2} \Sigma\right)$.

We can write the (A)SD condition

$\star \mathcal{R}_{b}^{a}= \pm \mathcal{R}_{b}^{a}$

as

$$
\begin{aligned}
\frac{d}{d t} h_{i j}(t) & =\frac{d}{d t} e_{i}^{a}(t) e_{j}^{b}(t) \delta_{a b} \\
& = \pm \star_{h} \mathcal{R}_{b}^{a}={ }^{(3)} R_{i j} \in \Gamma\left(\mathcal{T}^{0,2} \Sigma\right)
\end{aligned}
$$

where $\mathcal{R}_{b}^{a}=d w_{b}^{a}+w_{c}^{a} \wedge w_{b}^{c}$ is the curvature-form. Especially important to mention is, that we allow degenerate vierbeins because by construction the properties of the potential $k(\phi)$ associate positive lengths to negative scale factors. Concluding that, for our $k(\phi)$-model, we can make the statement that the associated Yang-Mills becomes a topological QFT, whose instantons $A^{ \pm}$are gradient flows between topological states with unbroken diffeomorphism invariance defined by the Chern-Simons states. The YM-field strength is

$$
\begin{aligned}
F_{i j}^{a \pm}= & {\left[D_{i}, D_{j}\right]=J_{a}^{+}\left[\partial_{i} A_{j}^{a+}-\partial_{j} A_{i}^{a+}\right.} \\
& \left.+\epsilon^{a b c}\left(A_{i b}^{-} A_{j c}^{+}+A_{i b}^{-} A_{j c}^{-}\right)\right] \\
& +J_{a}^{-}\left[\partial_{i} A_{j}^{a-}-\partial_{j} A_{i}^{a-}+\epsilon^{a b c} A_{i b}^{-} A_{j c}^{-}\right]
\end{aligned}
$$

and hence the 4-dimensional $S O$ (4)-YM-action $\int_{\mathrm{M}} \operatorname{Tr} F^{ \pm}$ $\wedge F^{ \pm}$is

$$
\begin{aligned}
S_{Y M}\left[A_{j}^{a \pm}\right]= & \int_{M} \epsilon^{i j k l}\left[\partial_{i} A_{j}^{a+}-\partial_{j} A_{i}^{a+}\right. \\
& \left.+\epsilon^{a b c}\left(A_{i b}^{-} A_{j c}^{+}+A_{i b}^{-} A_{j c}^{-}\right)\right] \\
& \times\left(\partial_{k} A_{a l}^{-}-\partial_{l} A_{a k}^{-}+\epsilon_{a b c} A_{k}^{b-} A_{l}^{c-}\right) .
\end{aligned}
$$

It is the gauge-equivalent to general relativity in fourdimensions if $k(\phi)=\dot{\phi}^{2}$. The action on the boundary follows as

$$
\begin{aligned}
& S_{C S}\left[A_{j}^{a \pm}\right] \\
& =\mathcal{N} \int_{\Sigma} e^{i j k}\left(A_{i a}^{+}\left(\partial_{j} A_{k}^{a-}-\partial_{k} A_{j}^{a-}+\epsilon_{a b c} A_{j}^{b-} A_{k}^{c-}\right)\right),
\end{aligned}
$$

which describes three-dimensional Einstein gravity with cosmological constant equivalently to (1.23). If we introduce the electric field $E_{i}^{a \pm}=F_{0 i}^{a \pm}$ with Poisson bracket

$$
\left\{A_{i}^{a \pm}(x), E_{j}^{b \pm}(y)\right\}=\mp M_{p}^{-2} \delta^{a b} \delta_{i j} \delta^{3}(y, x),
$$

then up to the Gauss constraint, the Hamiltonian of YMaction (2.17) in Euclidean signature reduces to

$H^{ \pm}=\int d^{3} x \operatorname{Tr}\left(\left(E_{i}^{a \pm}\right)^{2}-(\underbrace{\frac{1}{2} \epsilon_{i j k} F^{a j k \pm}}_{B_{i}^{a \pm}})^{2}\right)$.

$B_{i}^{a \pm}$ is the magnetic field. With the Dirac quantization procedure, the ground state

$$
\begin{aligned}
& \int_{\Sigma} d^{3} x \operatorname{Tr}\left(\frac{\delta}{\delta A_{i}^{a \pm}}+\frac{i}{2} e_{i j k} F^{a j k \pm}\right) \\
& \times\left(-\frac{\delta}{\delta A_{i}^{a \pm}}+\frac{i}{2} e_{i j k} F^{a j k \pm}\right) \Psi\left[A_{i}^{a \pm}\right]=0
\end{aligned}
$$

is given by the Euclidean Chern-Simons state

$\Psi_{0}\left[A_{i}^{a \pm}\right]=e^{ \pm i S_{C S}\left[A_{i}^{a \pm}\right]}$

because of the relation

$\frac{\delta S_{C S}\left[A_{i}^{a \pm}\right]}{\delta A_{i}^{a \pm}}=\epsilon^{i j k} F_{a j k}^{ \pm}=B_{a}^{i \pm}$.

Through

$$
\left(\frac{\delta}{\delta A_{i}^{a \pm}} \pm i B_{i}^{a \pm}\right) e^{ \pm i S_{C S}\left[A_{i}^{a \pm}\right]}=0 \rightarrow \pm i B_{i}^{a \pm}=E_{i}^{a \pm},
$$

which is always true for every metric whose scale factor is a solution of $k(\phi)=\dot{\phi}^{2}$, we define the second Chern-class as

$c_{2}\left[A_{i}^{a \pm}\right]=\int_{M} d^{4} x \operatorname{Tr} E_{i}^{a \pm} B_{i}^{a \pm}$

and hence

$S_{Y M}\left[A_{i}^{a \pm}\right]= \pm c_{2}\left[A_{i}^{a \pm}\right]$.

With this Ansatz and after introduction of the auxiliary $\mathfrak{s o}(4)$ valued 2-form $B_{\mu \nu}$, Yang-Mills action is

$S_{Y M}\left[A_{k}^{a \pm}\right]= \pm \int_{M} B^{a \pm} \wedge F_{a}^{ \pm}-\frac{1}{2} B^{a \pm} \wedge B_{a}^{ \pm}$,

from which we can read of the Poisson bracket

$$
\left\{B_{i j}^{a \pm}(x), A_{k}^{b \pm}(y)\right\}=\mp i M_{p}^{-2} \epsilon_{i j k} \delta^{a b} \delta^{3}(x-y) \text {. }
$$

Then follows immediately by replacing (2.19) with (2.28), that

$$
\begin{aligned}
& \left(F_{i j}^{a \pm}-B_{i j}^{a \pm}\right) \Psi_{0}\left[A_{i}^{a \pm}\right] \\
& =\left(F_{i j}^{a \pm}-i \epsilon_{i j k} \frac{\delta}{\delta A_{k}^{a \pm}}\right) \Psi\left[A_{i}^{a \pm}\right]=0
\end{aligned}
$$


is equivalent to

$$
\left(E_{i}^{a \pm} \mp B_{i}^{a \pm}\right) \Psi_{0}\left[A_{i}^{a \pm}\right]=\left(\frac{\delta}{\delta A_{i}^{a \pm}} \pm i B_{i}^{a \pm}\right) \Psi\left[A_{i}^{a \pm}\right]=0
$$

Since there are no degrees of freedom, the four-dimensional gauge-theory belonging to $k(\phi)$-theory is topological. Additionally, it is invariant under diffeomorphisms of the manifold $M$. To close the circle and recover General Relativity, we set

$B^{a \pm}=P_{\alpha \beta}^{a \pm} e^{\alpha} \wedge e^{\beta}=e^{0} \wedge e^{i} \pm e_{j k}^{i} e^{j} \wedge e^{k}$

where $P_{\alpha \beta}^{a \pm}$ is the projection operator onto the right and left-handed subalgebras $\mathfrak{s o ( 3 ) _ { R }}$ and $\mathfrak{s o}(3)_{L} \cdot B^{a \pm}$ are six $S O$ (3) 2-forms, divided into three self-dual and anti-selfdual fields. Together they provide an orthogonal basis of a six-dimensional space of 2-forms. Insertion of (2.31) into (2.27) leads to

$$
\begin{aligned}
S= & \pm \int_{M}\left(e^{0} \wedge e^{i} \pm e_{j k}^{i} e^{j} \wedge e^{k}\right) \wedge F_{a}^{ \pm} \\
& +\frac{1}{2} \epsilon_{\alpha \beta \gamma \delta} e^{\alpha} \wedge e^{\beta} \wedge e^{\gamma} \wedge e^{\delta} .
\end{aligned}
$$

Due to the split into self-dual and anti-self-dual parts, this can be written as

$S=\int_{M} \epsilon_{\alpha \beta \gamma \delta}\left(e^{\alpha} \wedge e^{\beta} \wedge F^{\gamma \delta \pm} \pm \frac{1}{2} e^{\alpha} \wedge e^{\beta} \wedge e^{\gamma} \wedge e^{\delta}\right)$

The above expression is equivalent to the Palatini-action. Varying with respect to the $S O(3)$ connections, the six equations of motion decompose into the (anti)-self-dual parts

$(D B)^{a \pm}=0$,

corresponding to the variation of the Palatini-action with respect to the $S O(4)$ connection

$\nabla e^{\alpha} \wedge e^{\beta}=0$.

Additionally, we have the Einstein equations

$\epsilon_{\alpha \beta \gamma \delta}(\underbrace{e^{\beta} \wedge F^{\gamma \delta \pm}(w)}_{=0} \pm \frac{1}{2} e^{\alpha} \wedge e^{\beta} \wedge e^{\gamma} \wedge e^{\delta})=0$. (2.36)

Since we are dealing with a manifold spacelike boundary, we need to add a boundary term to the action in order to have a well-defined variation principle. It turns out [20], that the Chern-Simons action pulled back to the boundary is a suitable candidate. The whole action in Euclidean signature is

$$
\begin{aligned}
S_{G R}\left[A^{ \pm}\right]= & \int_{M} B^{a \pm} \wedge F_{a}^{ \pm}-\frac{1}{2} B^{a \pm} \wedge B_{a}^{ \pm} \\
& \mp \frac{M_{p}^{2}}{32 \pi \sqrt{\Lambda}} \int_{\Sigma} \operatorname{Tr}
\end{aligned}
$$

$$
\times\left(A^{ \pm} \wedge d A^{ \pm}+\frac{2}{3} A^{ \pm} \wedge A^{ \pm} \wedge A^{ \pm}\right) .
$$

This means the families $h_{i j}(t)=( \pm \phi(t))^{2} \gamma_{i j} \in \Gamma\left(\mathcal{T}^{0,2} \Sigma\right)$ composed of the non-trivial (A)SD scale factors satisfying

${ }^{(3)} R_{i j}=2 \dot{\phi}^{2} \gamma_{i j}$

or equivalently

$\frac{\partial \phi}{\partial t}= \pm \sqrt{k(\phi)}$

follow trajectories in configuration space that are indeed gradients of the Chern-Simons invariant. The four-dimensional quantity that is foliated by $h_{i j}(t)$ is, for vanishing spacetime components, a gravitational instanton $g_{\mu \nu}$ with boundary. Different than e.g. in [9], the gradient flow is not unique, exhibited in form of the de-Sitter space. Rather it is part of a set of trajectories determined by the choice of the potential $k(\phi)$, upon which only the requirement of positive definiteness is made. As shown, the de-Sitter space is only a gradient flow trajectory of the CS-invariant if the potential is that of a harmonic oscillator, where the cosmological constant is a positive spring constant. The quantization of 3-dimensional CS-theory is well understood by path integral quantization [10], geometric quantization and the Reshetikhin-Turaev construction, which produces a topological QFT. This is a strong argument, that we can view the gravitational instantons of the $k(\phi)$-theory in the following way: As tunnelling processes between the critical loci of three-dimensional Einstein gravity defined by the action (2.2). Since we deal with FLRW-type metrics, we are even able to break the 4dimensional theory down to a one-dimensional of the scale factor. It is the reason, why we can do a similar simplification with the ill-defined path integrals of 4-dimensional quantum gravity, as it is shown below. We can reduce them to the partition functions of the systems defined by the one-dimensional action (1.9). The above identification is very intriguing, and we will return to it in the following (Table 1).

\section{Quantum $k(\phi)$-theory}

It is obvious that every semiclassical state describing solution of the $k(\phi)$-theory solves all constraints of quantum gravity simultaneously. Meaning, that all states realized by $k(\phi)$ theory are physical. We define the physical Hilbert space $\mathcal{H}_{\text {phy }}$ by imposing the first-class constraints in form of the operators $\widehat{\mathfrak{C}}$ as

$\widehat{\mathfrak{C}}\left|\Psi_{0, k(\phi)}\right\rangle=0$.

In other words: The infinite-dimensional kernel of all constraints $\left(\mathcal{H}_{p h y}\right)$ is completely determined by $k(\phi)$-theory. 
Table 1 Comparison between the most important expressions of Chern-Simons and $k(\phi)$-theory

\begin{tabular}{lll}
\hline 4d-action functional & $S_{g}\left[A_{i}^{a \pm}\right]= \pm S_{B F}+S_{C S}$ & $S_{g}\left[g_{\mu \nu}\right]=\int_{\Sigma \times \mathbb{R}} \sqrt{g}(4) R d^{4} x+\int_{\Sigma} 2 \varepsilon \sqrt{h} K d^{3} x$ \\
4-configuration & $A^{ \pm} \in \Omega^{1}(\Sigma \times \mathbb{R}, \mathfrak{g})$ & $g_{\mu \nu} \in \Gamma\left(\mathcal{T}^{0,2} M\right)$ \\
Equations of motion & $d_{A} \star F_{A^{ \pm}}=0$ & $R_{\mu \nu}=0$ \\
Variation $3 d$-action & $\delta S_{C S}=\int_{\Sigma}\left\langle\delta A^{ \pm} \wedge F_{A^{ \pm}}\right\rangle$ & $\delta S_{E H}=\int\left({ }^{(3)} R_{i j}-\frac{(3) R}{2} h_{i j}+\Lambda h_{i j}\right) \delta h^{i j} d^{3} x$ \\
Gradient flow & $\Omega^{1}(\Sigma, \mathfrak{g}) \ni \frac{d}{d t} A_{t}^{ \pm}= \pm \star h F_{A_{t}^{ \pm}}$ & $\frac{d}{d t} h_{i j}(t)={ }^{(3)} R_{i j} \in \Gamma\left(\mathcal{T}^{0,2} \Sigma\right)$ \\
Self-duality condition & $\star F_{A^{ \pm}}= \pm F_{A^{ \pm}}$ & $\star \mathcal{R}= \pm \mathcal{R}$ \\
3-configuration & $A_{t}^{ \pm} \in \Omega^{1}(\Sigma, \mathfrak{g})$ & $h_{i j}(t)=( \pm \phi(t))^{2} \gamma_{i j} \in \Gamma\left(\mathcal{T}^{0,2} \Sigma\right)$ \\
$3 d$-action functional & $S_{C S}\left[A^{ \pm}\right]=\frac{k}{4 \pi} \int_{\Sigma} \operatorname{Tr}\left(A^{ \pm} \wedge d A^{ \pm}+\frac{2}{3} A^{ \pm} \wedge A^{ \pm} \wedge A^{ \pm}\right)$ & $S_{E H}\left[h_{i j}\right]=\int_{\Sigma} d^{3} x \sqrt{h}\left({ }^{3)} R-2 \Lambda\right)$ \\
\hline
\end{tabular}

This may be written equivalently as

$\Psi_{0, k(\phi)}\left[A^{ \pm}\right]=e^{ \pm i S_{C S}\left[A^{ \pm}\right]}$.

The state is invariant under diffeomorphisms on $\Sigma$, small gauge transformations generated by the constraints as well as large gauge transformations due to its periodicity. Interestingly, it creates a notion of time in configuration space because it is always normal to the gauge orbits. Since we have chosen that $\Sigma$ has constant, positive scalar curvature, the homotopy class $\pi_{3}(\Sigma)=\mathbb{Z}$. We define a large gauge transformation for $n \in \mathbb{Z}$ as

$S_{C S}\left[A^{ \pm}\right] \rightarrow S_{C S}\left[A^{ \pm}\right]+n$.

This is nothing else than adding the action of the Yang-Mills instanton winding number $n$

$S_{C S}\left[A^{ \pm}\right] \rightarrow S_{C S}\left[A^{ \pm}\right]+\int_{S^{4}} \operatorname{Tr}\left(F_{A^{ \pm(n)}} \wedge F_{A^{ \pm(n)}}\right)$.

The circle compactification of the configuration space is therefore parametrized by the periodic function $S_{C S}[A]$ defining a dimensionless time. Furthermore, this means spacetimes in $\mathcal{H}_{\text {phy }}$ are trajectories in configuration space of connections $A_{i}^{a} \in \mathfrak{s u}(2)$. The instantons are corresponding to large gauge transformation requiring the $k$ in front of the CS-action to be an integer. Additionally, it indicates that the quantum field theories on the gravitational instantons defined through $k(\phi)$-theory satisfy the KMS condition and are therefore irreducible thermal. We may generalize the gauge transformations by incorporating large and small ones in Weyl-gauge

$\Psi_{k(\phi)}\left[A^{ \pm}\right] \rightarrow e^{i n \theta} \Psi_{k(\phi)}\left[A^{ \pm}\right]$

where $\theta$ is the Bloch momentum which arises from quantum tunnelling in configuration space. Hence, quantum states behave as Bloch waves in a periodic lattice and large gauge transformations can be interpreted as lattice translation. The last term in (3.4) is diffeomorphism invariant and independent of the metric as well as of local variations of the gauge potential. The topological invariant is called the ChernPontryagin index. In Euclidean Weyl-gauge it is related to the continued component of the Chern-Simons current

$$
\begin{aligned}
-\mathcal{P} & =\frac{1}{4 \pi^{2}} \int d^{4} x \partial_{\mu} K^{\mu}=\left.\frac{1}{4 \pi^{2}} \int d^{3} x K^{0}\right|_{x^{0}=\infty} \\
& =\frac{1}{24 \pi^{2}} \int d^{3} x \epsilon^{i j k} \operatorname{Tr} U^{-1} \partial_{i} U U^{-1} \partial_{i} U U^{-1} \partial U
\end{aligned}
$$

where $U \in G$. This means $S_{C S}[A]$ carries the phase response to the gauge transformation

$\Psi_{k(\phi)}\left[A^{ \pm}\right]=e^{ \pm i S_{C S}\left[A^{ \pm}\right] \theta} \Xi\left(A^{ \pm}\right)$.

where $\Xi(A)$ is invariant under the entire set of gauge transformation. In fact, we are dealing with a topological quantum field theory with the action in the bulk as

$S_{Y M}\left[A^{ \pm}, \theta\right]=\int d^{4} x \operatorname{Tr}\left(\frac{1}{2} F^{\mu \nu} F_{\mu \nu} \mp \frac{\theta}{16 \pi^{2}} \widetilde{F^{\mu \nu}} F_{\mu \nu}\right)$

where $\widetilde{F^{\mu \nu}}=\frac{1}{2} \epsilon^{\alpha \beta \mu \nu} F_{\alpha \beta}= \pm F^{\mu \nu}$ is the dual, that is defined only by the gauge group, which is $S O$ (4) = $S O(3)_{L} \oplus S O(3)_{R}$ and the $\theta$-angle. $\theta \in[0,2 \pi]$ determines the energy eigenvalues of the theory. The theta-term in the above Lagrangian is a total derivative and does neither contribute to the equations of motions nor to perturbation theory. However, it violates the CP-invariance of the original Lagrangian. The gauge equivalent vacuum gauge fields $A^{(n)}$ of the different sectors have topological charge zero and so it is possible to deform them continuously into each other before and after a gauge transformation. In general, however, this is not the case at the intermediate states of the deformation, which have larger expectation values than the vacuums at the beginning and the end of the gauge transformation. In the quantum gravity formulation, we gained in form of a topological QFT by starting with a particular $k(\phi)$ dependence, these "potential" barriers are literally scalarcurvature, and the vacuums are Ricci-flat manifolds. Thus, the instantons of quantum gravity mediate the vacuum tunnelling in form of the barrier penetration. The deformation process is to be taken literally, since from a classical viewpoint, the initial vacuum gauge field (Euclidean manifold) $\mathbb{R}_{-}^{3} \cong S_{\infty}^{3}$ at $x^{0}=-\infty$ becomes curved very slowly until 
it shrinks to $S_{l_{p}}^{3}$ at $x^{0}=0$, where it contracts in an "instant" by climbing over the potential barrier, reaching maximum curvature, before expanding similar abruptly to a nearly flat manifold on the other side of the barrier. After these rapid contracting and inflating processes in the vicinity of the penetration, the curvature asymptotically approaches zero, while the instanton reaches the final stage at $x^{0}=\infty$, which is the vacuum gauge field $\mathbb{R}_{+}^{3} \cong S_{\infty}^{3}$ in the next (previous) homotopic sector. Self-dual and anti-self-dual deformation processes "look" like one and the same gravitational instanton since their effect on the deformation is indistinguishable from one another. The deformation of a large gauge transformation has anything that we observe in our own expanding spacetime. It has inflation, explaining the flatness of space, however the curvature does not reach zero in finite times, so that it stays non-static. It solves the Big-bang singularity, since the barrier is of finite "height" (curvature). Quantum mechanically, as we already assumed, it is nothing else then the tunnelling of gravitons, which may be calculated perturbatively around the vacuum states. If we define the gauge transformation of the $n$-th class equivalently to (3.5) by

$\mathcal{U}^{(n)} \Psi_{k(\phi)}\left[A_{i}\right]=e^{i n \theta} \Psi_{k(\phi)}\left[A_{i}\right]$

then the theta vacuum reproduces the transformation law

$$
\begin{aligned}
\mathcal{U}^{(n)}|\theta\rangle & =\mathcal{N} \sum_{m=-\infty}^{\infty} e^{-i \theta m} \mathcal{U}^{(n)}|m\rangle \\
& =\mathcal{N} \sum_{m=-\infty}^{\infty} e^{-i m \theta}|m+n\rangle \\
& =\mathcal{N} \sum_{m=-\infty}^{\infty} e^{-i(k-n) \theta}|k\rangle \\
& =e^{i n \theta}|\theta\rangle,
\end{aligned}
$$

which is also true for every state build upon the vacuum. Thus, the vacuum structure of QG is a superposition of gaugeinvariant ground states, like in solid-state physics and QCD. The advantage of a topological quantum field theory is that it is background independent. As expected, for a theory of quantum gravity this means, the $n$-point functions are independent of the metric.

\section{One-dimensional QFT of the scale factor}

For now, we will return to the $\mathbb{Z}_{2}$-example. A complete description of the fluctuation-behaviour inside a double-well potential, would inquire a dilute instanton gas, which would resemble an infinitely contracting and expanding spacetime. The path integral of the action given in (1.2)

$\mathcal{J}\left[a_{i j}, b_{i j}\right] \sim \mathcal{N} \int \mathcal{D}\left[g_{\mu \nu}\right] e^{i S\left[g_{\mu \nu}\right]}$

$$
\begin{aligned}
= & \mathcal{N} \int \mathcal{D}\left[g_{\mu \nu}\right] \\
& \left.e^{\frac{i}{16 \pi l_{p}^{2}}\left(\int_{M} \sqrt{g}(4)\right.} R d^{4} x+\int_{\Sigma} 2 \varepsilon \sqrt{h} K d^{3} x\right),
\end{aligned}
$$

where we integrate over the boundaries of $g_{\mu \nu}$ at infinity and with $k(\phi)=\dot{\phi}^{2}$, corresponds to

$\mathcal{J}\left(\phi_{f}, \phi_{e}\right)=\mathcal{N} \int \mathcal{D} \phi e^{i S[\phi]}=\mathcal{N} \int D \phi e^{\frac{i}{2} \int d t\left(\partial_{t} \phi\right)^{2}+k(\phi)}$

since the scale factor is the only degree of freedom. In the case of non-trivial vacuum structures, the interpolating field configurations would be the saddle points of the well-defined Euclidean path integral

$\mathcal{J}\left(\phi_{f}, \phi_{e}\right)=\mathcal{N} \int \mathcal{D} \phi e^{-\frac{1}{2} \int d \tau\left(\partial_{\tau} \phi\right)^{2}+k(\phi)}$

where $\tau=i t$. In a more general way, the action (1.9) can be formulated in the setting of $\sigma$-Model

$S[\phi]=\int_{\varepsilon} d \tau \frac{1}{2} G_{i j} \dot{\phi}^{i} \dot{\phi}^{j}+V(\phi)$.

in Euclidean time, where $\phi: \varepsilon \rightarrow N . G_{i j}$ is the pullback to $\varepsilon$ of the hyperbolic metric on $(N, G)$ and $2 V(\phi)=k(\phi)$. This means we view the map $\phi(\tau)$ as the trajectory of a particle in the space $N$ with local coordinates $\phi^{\alpha}, \alpha=1, \ldots, n$ , which is the configuration space of scale factors of spatial, maximally symmetric pseudo-Riemannian metrics. We denote the configuration space of the system (4.4) as $\mathcal{U}=$ $\{\phi(\tau) \in \operatorname{Maps}(\mathbb{R}) / E[\phi]<+\infty\}$. In case of a non-trivial vacuum structure, $\mathcal{U}$ is non-connected and kinks form equipotential curves inside it, which results in the occurrence of zero modes due to the translational invariance of the action, while trivial solutions are points in $\mathcal{U}$. One should keep in mind however, that those trivial solutions correspond to three-dimensional Euclidean manifolds, whereas interpolating solutions are bounded by the trivial solutions and correspond to a family of boundary metrics with non-zero curvature labelled by the flow parameter $t$. Basically, the procedure is to associate points in $N$ to an interval of $\varepsilon$ parametrized through $i t=\tau$ by means of minimizing the action (4.4). These "worldlines" are formed in such a way that they lie in $\mathcal{U}$. Since the minima of the positive definite potential $V(\phi) \sim k(\phi)$ are by construction scale factors of Ricci-flat manifolds $(\Sigma, h)$, and the requirement for a non-trivial finite energy solution to approach them asymptotically in order to minimize the energy in its sector of configuration space $\mathcal{U}$, the dynamic of a classical spacetime can be imagined as propagating towards vanishing curvature. Although "propagating" is wrong in the sense, that every hypersurface might exist next to each other in timelessness, whose alignment is determined by the dynamics of the scale factor inside $N$, determined in return by the structure of the potential $k(\phi)$, 
and only the experiencing of different imprints of our brains on the hypersurfaces according to causality principles creates the illusion of time or the flowing of space towards minimum curvature, respectively. This also means that constant Riccicurvature of the hyperslices is less an intrinsic characteristic, rather than a simple potential force on the image $\phi(\varepsilon) \subset N$. Upon variation of the action (4.4) the equations of motion unfold to be

$\frac{d^{2} \phi^{i}}{d \tau^{2}}+\Gamma_{j k}^{i} \dot{\phi}^{j} \dot{\phi}^{k}=G^{i j} \frac{d V}{d \phi^{j}}$

where $\Gamma_{j k}^{i}$ is the Levi-Civita connection on $N$ pulled back to $\varepsilon$. Equation (4.5) reduces to (1.11) or (1.8) respectively, if $G_{i j}=\delta_{i j}$. For $G_{i j}=\eta_{i j}$ we'd get a minus sign. Note that the boundary term of $\varepsilon$ is the tautological one-form on configuration space $N$. Equation (4.5) states that for a vanishing potential the particle "flows" along geodesics in $(N, G)$. Similarly, we could have used the one-dimensional ChernSimons action functional associated to the invariant polynomial corresponding to the first Chern class, to formulate the one-dimensional action. In this case we view the configuration space $N$ as the space of $\mathfrak{g}$-valued one forms $\Omega^{1}(\varepsilon, \mathfrak{g})$, which is just the space of $\mathfrak{u}(n)$-valued functions because $\varepsilon \subset \mathbb{R}$. According to one-dimensional QFT, the Hamilton operator $H: L^{2}(N) \rightarrow L^{2}(N)$ on $(N, G)$ is defined as

$\widehat{\mathcal{H}}=-\frac{1}{2} \frac{1}{\sqrt{G}} \frac{\partial}{\partial \phi^{i}}\left(\sqrt{G} G^{i j} \frac{\partial}{\partial \phi^{j}}\right)+V=-\frac{1}{2}[\Delta-k(\phi)]$.

A $\Psi \in L^{2}(N)$ is acted on by $\widehat{\mathcal{H}}$ such that

$\widehat{\mathcal{H}} \Psi(\phi(\tau))=0$.

Therefore, the constraint (4.7) is the scale factor analogue to the Wheeler-De-Witt equation,

$$
\left(-G^{i j k l} \frac{\delta}{\delta h^{i j}} \frac{\delta}{\delta h^{k l}}-\sqrt{h^{(3)}} R\right) \Psi\left[h_{i j}\right]=0
$$

which can be also derived by integration of the Hamilton constraint of canonical quantum gravity over the 3-surface. (The momentum constraint is satisfied automatically.) It can in return be formulated equally in a representation, where the field is a section of a spin-bundle

$$
\left(-\frac{\delta}{\delta A_{i}^{a \pm}} \frac{\delta}{\delta A_{i}^{a \pm}}+e_{i j k} F^{a j k \pm}\right) \Psi[A]=0 .
$$

We saw in the previous section, that solutions to this equation are given through the gravitational instantons of $k(\phi)$-theory or in terms of the Jones-polynomial of a knot $K$ in $\Sigma$ [11]

$J(q)=\int \operatorname{trhol}_{A}(K) e^{i S_{C S}[A]}$.

Representation and gauge group can be modified. The wavefunction $\Psi$ in (4.7) depends only one the scale factor of $h_{i j}$.
The probability for the scale factor $\phi$ to propagate from an initial position $\phi_{e} \in \mathrm{N}$ to a final position $\phi_{f} \in \mathrm{N}$ is encoded by the propagator $\mathcal{J}\left(\phi_{f}, \phi_{e}\right) \in \varepsilon \subset \mathrm{N} \times \mathrm{N}$, which satisfies

$$
\begin{aligned}
\widehat{\mathcal{H}} \mathcal{J}\left(\phi_{f}, \phi_{e}\right) & =-\frac{\partial}{\partial \mathrm{t}} \mathcal{J}\left(\phi_{f}, \phi_{e}\right) \\
\mathcal{J}_{0}\left(\phi_{f}, \phi_{e}\right) & =\delta\left(\phi_{f}-\phi_{e}\right)
\end{aligned}
$$

where $\delta \in \mathrm{N} \subset \mathrm{N} \times \mathrm{N}$. Therefore, $\mathcal{J}\left(\phi_{f}, \phi_{e}\right)$ indeed can be calculated from

$\mathcal{J}\left(\phi_{f}, \phi_{e}\right)=\int \mathcal{D} \phi e^{-\int_{C} d \tau \frac{1}{2} G_{i j} \dot{\phi}^{i} \dot{\phi}^{j}+V(\phi)}$.

In the CS-formulation of one-dimensional QFT we could have used Wilson loops if we demand that the one-dimensional manifold is a circle. Then the Wilson loop for $\phi \in$ $\Omega^{1}(\varepsilon, \mathfrak{g})[12]$

$W_{S^{1}}^{r}(\phi)=\operatorname{hol}_{S^{1}}^{r}(\phi)$

is proportional to the path integral of the one-dimensional CS-theory, if the moduli space is a coadjoint orbit of the gauge group and if the irreducible representation $r$ is defined via the orbit method [13]. From this perspective it is even more clear that the scale factors behave like topological particles influenced by the background field defined by the onedimensional CS-theory or equally by the $\sigma$-Model (4.4). Thus, through the perspective of $\phi$ as a trajectory inside $k(\phi)$, we can calculate solutions to the Wheeler-De-Witt equation for the metrics of the form (1.2). We stress, that the $t$ is only used to parametrize the "worldline", which is the one-dimensional manifold $\varepsilon$, or more precisely the image $\phi(\varepsilon) \subset N$, and it should not be confused with the physical time of ordinary quantum mechanics, labelling the evolution of particle-waves inside space rather than the propagation of space itself through superspace. Furthermore, the n-point correlation function of local operators $\widehat{\mathcal{A}} \in L^{2}(N)$ corresponding to real-valued functions $a: N \rightarrow \mathbb{R}$ is

$$
\left\langle\phi_{f} \mid \mathcal{T}\left\{\prod_{i} \widehat{\mathcal{A}}\left(t_{i}\right)\right\} \phi_{i}\right\rangle=\int_{\mathcal{C}\left[\phi_{f}, \phi_{e}\right]} \mathcal{D} \phi e^{-S[\phi]} \prod_{i=1}^{n} A_{i}\left(\phi\left(t_{i}\right)\right)
$$

with $\mathcal{C}\left[\phi_{f}, \phi_{e}\right]$ the field of maps $\phi:\left[T_{1}, T_{2}\right] \rightarrow N$ with $\phi_{f}=\phi\left(T_{2}\right) \quad \phi_{e}=\phi\left(T_{1}\right)$. The $A_{i}\left(\phi\left(t_{i}\right)\right)$ are just functions evaluated at the points $\phi\left(t_{i}\right) \in N$, whereas the insertion times of the local operators $\widehat{\mathcal{A}}$, acting at just one point of the scale factor or on a single hypersurface equivalently, must be ordered. For example, that $k(\phi)$ is given as in (1.12) the tunnelling-propagator of the system is

$$
\mathcal{J}(1,-1)=\sqrt{\frac{2 M_{p}}{\pi}} e^{-M_{p} \tau} \sinh \left(4 \tau \sqrt{\frac{2 M_{p}^{\frac{3}{4}}}{\pi}} e^{-\frac{4 M_{p}}{3}}\right)
$$


$\mathcal{J}(-1,-1)=\sqrt{\frac{2 M_{p}}{\pi}} e^{-M_{p} \tau} \cosh \left(4 \tau \sqrt{\frac{2 M_{p}^{\frac{3}{4}}}{\pi}} e^{-\frac{4 M_{p}}{3}}\right)$.

It involves the second order fluctuations in the vacuum- and kink-sector [14-17]. For a detailed description and calculation of (4.14) for the double-well potential we refer to [18] and for further investigation in terms of ADS/CFT is provided by [18-20].

Acknowledgements We acknowledge support from Leipzig University for Open Access Publishing.

Data Availability Statement This manuscript has no associated data or the data will not be deposited. [Authors' comment: All the ideas presented in this paper are of completely theoretical nature. Therefore there is no associated data.]

Open Access This article is licensed under a Creative Commons Attribution 4.0 International License, which permits use, sharing, adaptation, distribution and reproduction in any medium or format, as long as you give appropriate credit to the original author(s) and the source, provide a link to the Creative Commons licence, and indicate if changes were made. The images or other third party material in this article are included in the article's Creative Commons licence, unless indicated otherwise in a credit line to the material. If material is not included in the article's Creative Commons licence and your intended use is not permitted by statutory regulation or exceeds the permitted use, you will need to obtain permission directly from the copyright holder. To view a copy of this licence, visit http://creativecomm ons.org/licenses/by/4.0/.

Funded by SCOAP ${ }^{3}$.

\section{References}

1. J. J. Oh, H. S. Yang, Einstein Manifolds as Yang-Mills Instantons. arXiv:1101.5185 (2011)
2. R. Rajaraman, Solitons and Instantons (North-Holland, Amsterdam, 1987)

3. E.B. Bogomolny, The stability of classical solutions. Sov. J. Nucl. Phys. 24, 449 (1976)

4. N. Manton, P. Sutcliffe, Topolical Solitons (Cambridge, 2004)

5. V. Venkatesh, D. Mallesha Naik, A. Kumara H, *-Ricci solitons and gradient almost *-Ricci solitons on Kenmotsu manifolds (2019). arXiv: $1901.05222 \mathrm{v} 1$

6. E. Witten, Three-dimensional gravity revisited (2007). arXiv:0706.3359

7. S. Donaldson, Floer Homology Groups in Yang-Mills Theory. Cambridge Tracts in Mathematics, vol. 147 (2002)

8. A. Floer, An instanton-invariant for 3-manifolds. Commun. Math. Phys. 118(2), 215-240 (1988)

9. L. Smolin, Quantum gravity with a positive cosmological constant (2002). arXiv:hep-th/0209079

10. J. Andersen, H. Boden, A. Hahn, B. Himpel (eds.) Chern-Simons Gauge Theory: 20 Years After. AMS/IP Studies in Advanced Mathematics, vol. 50 (AMS, Providence, RI, 2011). arXiv:0911.2687

11. E. Witten, Quantum field theory and the Jones polynomial. Commun. Math. Phys. 121(3), 351-399 (1989)

12. C. Beasley, Localization for Wilson Loops in Chern-Simons Theory, (2010). arXiv:0911.2687 [hep-th]

13. A.P. Balachandran, S. Borchardt, A. Stern, Lagrangian and Hamiltonian descriptions of Yang-Mills particles. Phys. Rev. D 17, 3247$3256(1978)$

14. E. B. Bogomolny, The stability of classical solutions, Sov. J. Nucl. Phys. 24, 449 (1976)

15. D.M.A. Stuart, Hamilton quantization of solitons in the $\phi_{1+1}^{4}$ quantum field theory (2019). arXiv:1904.02588

16. L.D. Faddeev, V.E. Korepin, Quantum theory of solitons. Phys. Rep. 42C, 1-87 (1978)

17. S.F. Coleman, Classical Lumps and Their Quantum Descendants, 1975 Erice Lectures, in Aspects of Symmetry (Cambridge University Press, New York, 1985)

18. H. Forkel, A primer on instantons in QCD (2002). arXiv:hep-ph/0009136v2

19. A. Vilenkin, M. Yamada, The tunneling wave function of the Universe. Phys. Rev. D 98(6), 066003 (2018)

20. G. Conti, Holographic Wavefunctions of the Universe (Belgium, Leuven, 2017) 